\title{
Curcumin played an anti-cancer role in colorectal cancer via mediating circ_KIAA1199-related regulatory axis
}

\author{
Xue-Feng SUN ${ }^{1}$, Liang $\mathrm{LI}^{1}$, Yan-Bing LIU ${ }^{1 *}$ (D)
}

\begin{abstract}
Curcumin has been found to have anti-cancer role in many cancer, including colorectal cancer (CRC). However, it is not clear whether curcumin can be involved in CRC progression by mediating the circRNA regulatory axis. Curcumin inhibited the proliferation, migration, invasion, and promoted the apoptosis of CRC cells. Circ_KIAA1199 was an upregulated circRNA in $\mathrm{CRC}$, and its overexpression could reverse the negative regulation of curcumin on CRC progression. MiR-433-3p was found to be sponged by circ_KIAA1199, and its mimic abolished the regulation of circ_KIAA1199 overexpression on the progression of curcumin-treated CRC cells. ADAM10 was a target of miR-433-3p and its expression was regulated by circ_KIAA1199 and curcumin. The suppressive effect of miR-433-3p inhibitor on the progression of curcumin-treated CRC cells could be reversed by ADAM10 silencing. Also, curcumin reduced CRC tumor growth by regulating the circ_KIAA1199/miR-433-3p/ADAM10 axis in vivo. Our data showed that curcumin could restrain CRC progression, which was achieved through the circ_KIAA1199/ miR-433-3p/ADAM10 pathway.
\end{abstract}

Keywords: colorectal cancer; curcumin; circ_KIAA1199; miR-433-3p; ADAM10.

Practical Application: Identifying the molecular mechanism by which curcumin inhibits the progression of CRC may provide a potential molecular target for the treatment of CRC.

\section{Introduction}

Colorectal cancer (CRC) refers to tumors caused by malignant lesions of the colorectal mucosa under the action of various carcinogenic factors such as environment or genetics (Brody, 2015; Weitz et al., 2005). As a common malignant tumor of the digestive tract, CRC reduces the quality of life of patients and imposes a great financial burden on patients (Patel \& Ahnen, 2018). Although many treatments such as surgery and chemoradiotherapy have made many efforts to improve the survival rate of CRC patients, the prognosis is still not satisfactory (Sheng et al., 2018; Wang et al., 2019). In recent years, the anti-cancer effect of traditional Chinese medicine (TCM) extracts has received great attention (Danciu, 2020; Li et al., 2016). Curcumin is one of the main active ingredients in the TCM turmeric, which has been proven to have various pharmacological effects such as anti-inflammatory and anti-oxidant (Kotha \& Luthria, 2019; Nelson et al., 2017). With the deepening of research, curcumin is found to have a wide range of anti-cancer effects in many cancers including CRC (Pricci et al., 2020; Unlu et al., 2016). Therefore, elucidating the potential molecular mechanism of curcumin regulating CRC progression can help us better understand the anti-cancer function of curcumin.

Circular RNA (circRNA), a non-coding RNA with a special covalent closed loop structure, has been found to be abnormally expressed in human diseases (Zhang et al., 2018c). There is increasing evidence that circRNA is involved in the malignant progression of cancer and can be used as potential therapeutic targets for cancer (Lei et al., 2019; Zhang et al., 2018a). In CRC- related researches, it has been confirmed that many circRNAs are related to CRC progression, such as hsa_circ_000166 (Hao \& Zhang, 2020), hsa_circ_002144 (Wu et al., 2020) and hsa_circ_0000392 (Xu et al., 2020). In past studies, Tian et al. (2019) discovered that hsa_circ_0004585 (derived from KIAA family 1199 (KIAA1199) genic, also called circ_KIAA1199) was upregulated in CRC tissues and the peripheral blood of patients, and could be used as a potential diagnostic marker for CRC (Tian et al., 2019). However, it is not clear whether circ_KIAA1199 was involved in regulating the progression of CRC.

The endogenous competitive RNA (ceRNA) mechanism of circRNA reveals that circRNA can be served as the ceRNA of microRNA (miRNA) to indirectly regulate downstream gene expression (Song et al., 2020; Wu et al., 2019). Our study showed that curcumin could regulate circ_KIAA1199 expression, so we speculated that curcumin might mediate the progression of CRC by regulating circ_KIAA1199. In addition, we also revealed the molecular mechanism of curcumin's anti-cancer effect through the hypothesis of circRNA/miRNA/mRNA axis.

\section{Materials and methods}

\subsection{Cell culture and curcumin treatment}

Human CRC cells (HCT116 and SW480) and normal colorectal mucosa cells (FHC) (ATCC, Manassas, VA, USA) were cultured in RPMI-1640 medium (containing double antibiotics; 
Solarbio, Beijing, China) containing 10\% fetal bovine serum (FBS; Gibco, Waltham, MA, USA) at $37^{\circ} \mathrm{C}$ with $5 \% \mathrm{CO}_{2}$. Curcumin (Sigma-Aldrich, St. Louis, MO, USA) was diluted to different concentrations $(5,10$, and $20 \mu \mathrm{M})$ with DMSO (Sigma-Aldrich). Different concentrations of curcumin were used to treat CRC cells for $48 \mathrm{~h}$.

\subsection{Colony formation assay}

CRC cells were seeded into 6-well plates (150 cells/well). After culturing for 2 weeks, the cells were fixed with $4 \%$ paraformaldehyde (Solarbio) and stained with $0.5 \%$ crystal violet (Solarbio). The number of colonies (> 50 cells) were counted under a microscope (Nikon, Tokyo, Japan).

\subsection{MTT assay}

HCT116 and SW480 cells were seeded into 96-well plates $\left(1 \times 10^{3}\right.$ cells/well). After the cells attached to the wells, MTT solution (Sangon, Shanghai, China) was added into cells at the indicated time points for $4 \mathrm{~h}$, following by incubating with Formazan Solubilization Solution (Sangon) for $10 \mathrm{~min}$. The optical density (OD) value at $570 \mathrm{~nm}$ was measured by the microplate reader (Bio-Rad, Hercules, CA, USA).

\subsection{Transwell assay}

Transwell chamber $(8.0 \mu \mathrm{m}$; BD Biosciences, Franklin Lakes, NJ, USA) was used to detect cell migration and invasion ability. It should be noted that for the detection of invasion, the upper chamber needed to be pre-coated with a Matrigel (BD Biosciences). HCT116 and SW480 cells suspended with RPMI1640 medium were seeded into the upper chambers, and the lower chambers were filled with RPMI-1640 medium and 10\% FBS. After $24 \mathrm{~h}$, the cells on the lower surface were fixed and stained to count the numbers of migrated and invaded cells under a microscope $(100 \times$; Nikon $)$.

\subsection{Flow cytometry}

The Annexin V-FITC Apoptosis Detection Kit (SigmaAldrich) was used to detect apoptotic cells by flow cytometry. CRC cells were collected and re-suspend with $1 \times$ Binding Buffer. After staining with Annexin V-FITC and Propidium Iodide Solution for $10 \mathrm{~min}$, the cells were subjected to flow cytometer (BD Biosciences) to analyze cell apoptotic rate (\%).

\subsection{Clinical specimens}

A total of 43 patients with CRC who undergoing routine surgery were enrolled from Chongqing University Three Gorges Hospital. CRC tumor tissues and the corresponding adjacent normal tissues were stored at $-80^{\circ} \mathrm{C}$. This study was approved by the Ethical Committee of Chongqing University Three Gorges Hospital, and informed consent was obtained from all patients.

\subsection{Quantitative real-time PCR (qRT-PCR)}

RNAiso Plus (Takara, Tokyo, Japan) was utilized to isolate total RNA from tissues and cells. After the RNA was reversely transcribed into cDNA using PrimerScript RT Reagent Kit (Takara), the qRT-PCR was conducted using SYBR Green PCR Master Mix (Bio-Rad). GAPDH and U6 were used as internal controls and the results was presented by $2^{-\Delta \Delta \mathrm{Ct}}$ method. The primers were shown as below: circ_KIAA1199, F 5'-GACCGTGACAGCAAGATGTG-3', R 5'-GGGAAGCAGGTCAGAGTGAG-3'; KIAA1199, F 5'-CACGGTCTAT TCCATCCACATC-3', R 5'-GGTTCGCAAAACAATCGGCT-3'; miR-4333p, F 5'-GCCGAGATCATGATGGGCTCCT-3', R 5'-ATCCAGTGCAGGGTCCGAGG-3';

A disintegrin and metalloproteinase 10 (ADAM10), F 5'-ATGGGAGGTCAGTATGGGAATC-3', R 5'-ACTGCTCTTTTGGCACGCT-3'; GAPDH, F 5' - C TCTGCTCCTCCTG T TCGAC-3', R 5' - C G A C C A A A T C C G T T G A C T C C - 3'; U 6, F 5'-GCTTCGGCAGCACATATACTAAAAT-3', R 5'-CGCTTCACGAATTTGCGTGTCA-3'.

\subsection{Subcellular localization assay}

Nuclear and cytoplasmic RNA of CRC cells were separately isolated using PARIS Kit (Invitrogen, Carlsbad, CA, USA). QRT-PCR was used to analyze the circ_KIAA1199, GAPDH and U6 expression in the nucleus and cytoplasm of HCT116 and SW480 cells.

\subsection{Actinomycin D (ActD) assay}

CRC cells were treated with ActD solution (Sigma-Aldrich) for $1 \mathrm{~h}$. After the cells were further cultured for indicated times $(0,8,16$ and $24 \mathrm{~h})$, the expression of circ_KIAA1199 and linear KIAA1199 mRNA was examined with qRT-PCR.

\subsection{Cell transfection}

The vectors and oligonucleotides were obtained from GenePharma (Shanghai, China) and were transfected into cells using Lipofectamine 3000 reagent (Invitrogen). The vectors included circ_KIAA1199 pCD-ciR overexpression vector and lentiviral overexpression vector (circ-KIAA1199 and lenti-circ_KIAA1199) or their controls (vector and lenti-NC). The oligonucleotides included miR-433-3p mimic and inhibitor (miR-433-3p and anti-miR-433-3p) or their scrambled controls (miR-NC and anti-miR-NC), ADAM10 small interference RNA (si-ADAM10) and its negative control (si-NC).

\subsection{Western Blot (WB) analysis}

Total protein was extracted using RIP Assay Kit (Solarbio), and its concentration was quantified using BCA Kit (Beyotime, Shanghai, China). Then, the protein was separated by $10 \%$ SDSPAGE gel and transferred to PVDF membrane (Beyotime). The membrane was blocked with $5 \%$ skimmed milk for $1 \mathrm{~h}$, and then treated with anti-Bcl-2 (1:500, 100126-T36, Sino Biological, Beijing, China), anti-Bax (1:2,000, 106076-T32, Sino Biological), anti-ADAM10 (1:10,000, 310235-T34, Sino Biological) or anti-GAPDH (1:2,000, 10094-T52, Sino Biological) overnight at $4{ }^{\circ} \mathrm{C}$. The membrane was incubated with HRP-labeled Goat anti-Rabbit $\operatorname{IgG}(\mathrm{H}+\mathrm{L})(1: 1,000, \mathrm{~A} 0208$, Beyotime) for $2 \mathrm{~h}$. The membrane was finally reacted with BeyoECL Moon ECL chemiluminescence Kit (Beyotime) to detect protein bands. 


\subsection{Dual-luciferase reporter assay}

The binding or mutated sequences of circ_KIAA1199 and ADAM10 3'UTR with miR-433-3p were inserted into the pmirGLO-REPORT reporter vector (Promega, Fitchburg, WI, USA) to construct the corresponding wild-type (WT) or mutant type (MUT) vectors, respectively. HCT116 and SW480 cells in 24-well plates were co-transfected with the reporter vectors and miR-433-3p mimic or miR-NC. Relative luciferase activity was detected using Dual-Luciferase Reporter Assay Kit (Vazyme, Nanjing, China).

\subsection{Mice xenograft models}

Twelve male BALB/c nude mice (Vital River, Beijing, China) were divided into 4 groups $(n=3) .2$ groups were subcutaneously injected with HCT116 cells $\left(2 \times 10^{6}\right)$ and then intraperitoneally injected with $50 \mathrm{mg} / \mathrm{kg}$ curcumin or the same amount of DMSO (as control) every day. The other 2 groups were subcutaneously injected with SW620 cells transfected with lenti-NC or lenticirc_KIAA1199 and then intraperitoneally injected with curcumin every day. After 10 days, the tumor width (W) and length (L) were measured every 5 days to calculate tumor volume $\left(\mathrm{L} \times \mathrm{W}^{2} / 2\right)$. After 30 days, the mice were euthanized and the tumors were removed for other experiments. Our animal study was approved by the Animal Ethical Committee of Chongqing University Three Gorges Hospital.

\subsection{Immunohistochemistry (IHC) staining}

The paraffin-embedded sections of mice tumor tissues were dewaxed and rehydrated before treatment with 3\% hydrogen peroxide solution (Fusheng Biotechnology, Shanghai, China) for $30 \mathrm{~min}$. The sections were incubated with anti-Ki67 (1:100, SAB4501880, Sigma-Aldrich) or anti-Cleaved caspase3 (1:100, SAB1305630, Sigma-Aldrich) for $24 \mathrm{~h}$, followed by hatching with HRP-labeled Goat anti-Rabbit IgG (H+L) (1:1,000, A0208, Beyotime). After that, the sections were stained with hematoxylin (Sigma-Aldrich). The Ki67 and Cleaved caspase3 positive cells were observed under a microscope $(40 \times$; Nikon).

\subsection{Statistical analysis}

The GraphPad Prism 7 software (GraphPad, La Jolla, CA, USA) was used for statistical analysis. The data were presented as mean \pm standard deviation. The comparisons were analyzed using Student's $t$-test or analysis of variance with Tukey's post-hoc test. Pearson correlation analysis was performed to analyze the correlations among circ_KIAA1199, miR-433-3p and ADAM10. $P<0.05$ indicated a statistically significant.

\section{Results}

\subsection{Curcumin inhibited proliferation, migration, invasion and enhanced apoptosis in CRC cells}

To confirm the effect of curcumin on the progression of CRC and to screen the optimal treatment concentration of curcumin, HCT116 and SW 480 cells were treated with different concentrations $(5,10$, and $20 \mu \mathrm{M})$ of curcumin. After curcumin treatment, we found that the number of colonies and the viability of HCT116 and SW480 cells were markedly reduced in a concentration-dependent manner (Figure 1A-C). Not only that, the migration and invasion abilities of HCT116 and SW480 cells were decreased significantly with the increase of curcumin concentration (Figure 1D, E). The results of flow cytometry showed that curcumin treatment could significantly promote the apoptotic rate of HCT116 and SW480 cells, and the higher the concentration of curcumin, the higher the cell apoptosis (Figure 1F). Since the suppressive effect of $20 \mu \mathrm{M}$ curcumin on CRC progression was superior to other concentrations, the treatment concentration of curcumin in subsequent experiments was $20 \mu \mathrm{M}$.

\subsection{Curcumin reduced the expression of circ_KIAA1199, an upregulated circRNA in CRC tissues and cells}

In the CRC tumor tissues, we discovered that circ_KIAA1199 was remarkably higher than in adjacent normal tissues (Figure 2A). Besides, the expression of circ_KIAA1199 also was upregulated in HCT116 and SW480 cells compared with that in FHC cells (Figure 2B). To our delight, in HCT116 and SW480 cells treated with different concentrations of curcumin, circ_KIAA1199 expression was significantly decreased in a concentration-dependent manner (Figure 2C). Using the subcellular localization assay, we confirmed that circ_KIAA1199 was mainly distributed in the cytoplasm (Figure 2D, E), suggesting that circ_KIAA1199 was mainly involved in post-transcriptional regulation. In addition, ActD assay showed that circ_KIAA1199 was much more stable than its linear RNA KIAA1199 (Figure 2F, G), confirming that circ_KIAA1199 was indeed a circRNA. These data showed that circ_KIAA1199 was an upregulated circRNA in CRC and its expression was regulated by curcumin.

\subsection{Circ_KIAA1199 overexpression relieved the inhibition of curcumin on CRC progression}

In order to confirm that curcumin regulated CRC progression by mediating circ_KIAA1199, we constructed the circ_KIAA1199 overexpression vector for functional verification. First, we determined that the circ_KIAA1199 overexpression vector could indeed promote circ_KIAA1199 expression without affecting linear KIAA1199 mRNA expression (Figure 3A, B). After the transfected HCT116 and SW480 cells were treated with curcumin, we found that the suppressive effects of curcumin on the number of colonies and cell viability could be abolished by circ_KIAA1199 overexpression (Figure 3C-E). Also, overexpressed circ_KIAA1199 could overturn the inhibiting regulation of curcumin on the migration and invasion of HCT116 and SW480 cells, and the promoting effect on cell apoptosis (Figure 3F-H). Additionally, the detection results of apoptosis markers (Bcl-2 and Bax) showed that curcumin could decrease $\mathrm{Bcl}-2$ protein expression and promote Bax protein expression in HCT116 and SW480 cells, while these effects could be reversed by overexpressing circ_KIAA1199 (Figure 3I-J). Therefore, we confirmed that curcumin hindered CRC progression by regulating circ_KIAA1199. 

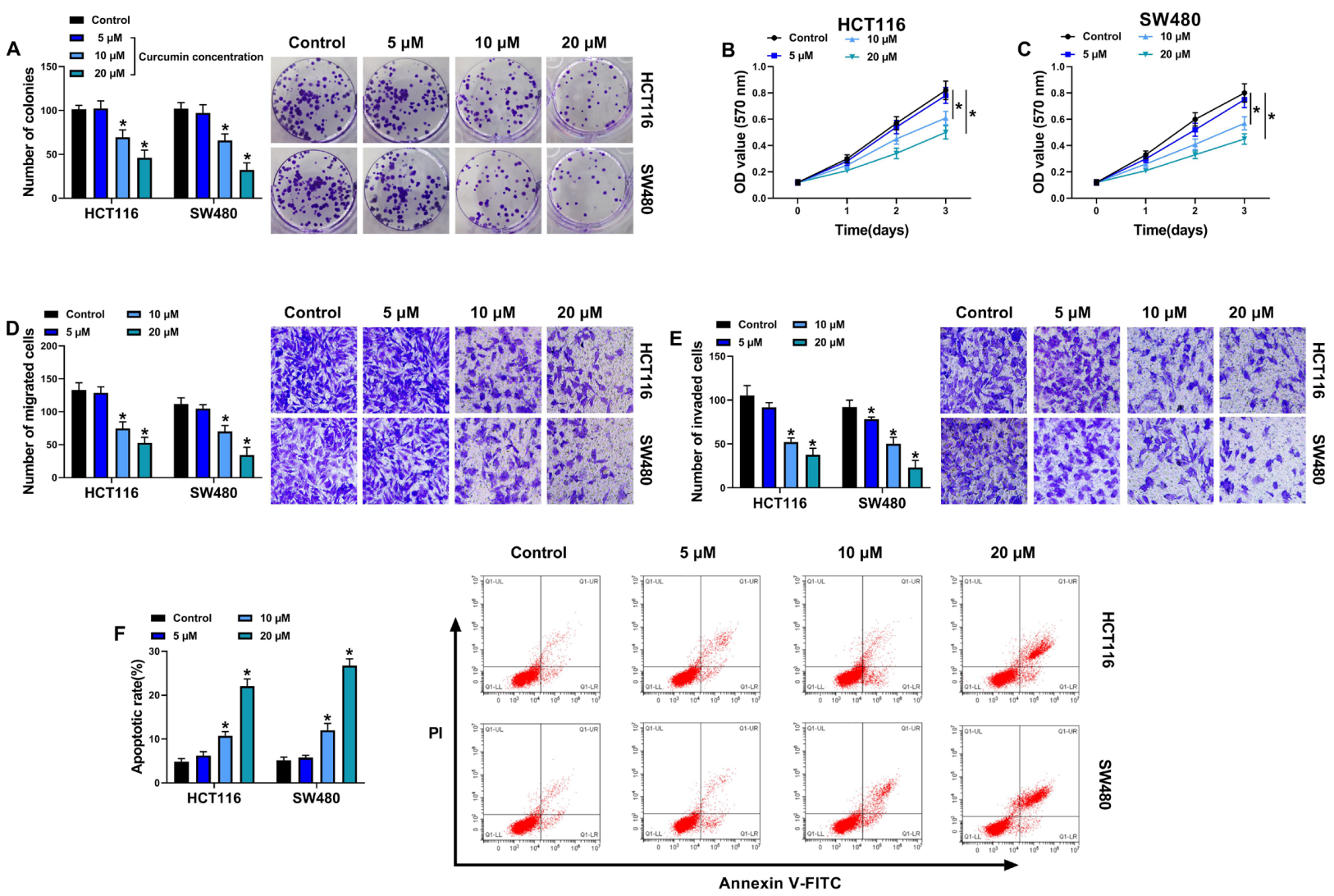

Figure 1. Curcumin inhibited CRC cell progression. HCT116 and SW480 cells were treated with different concentrations (5, 10, and $20 \mu \mathrm{M})$ of curcumin. Non-treated cells were used as control. (A) The number of colonies was measured using colony formation assay. (B-C) The cell viability was detected by MTT assay. (D-E) The numbers of migrated and invaded cells were determined using transwell assay. (F) Flow cytometry was used to test the apoptotic rate of cells. ${ }^{\star} P<0.05$.
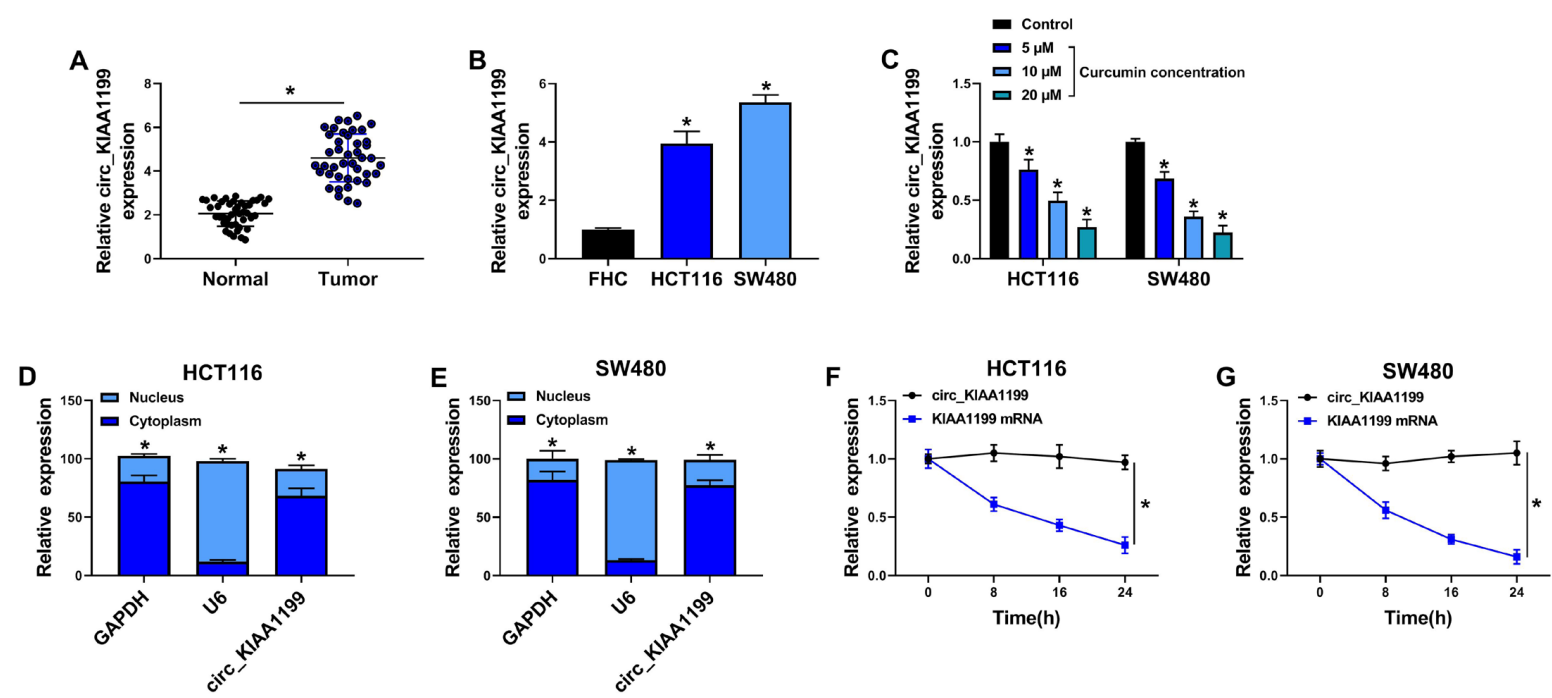

Figure 2. The expression of circ_KIAA1199 in CRC tissues and cells. (A) Circ_KIAA1199 expression in CRC tumor tissues (Tumor) and adjacent normal tissues (Normal) was examined using qRT-PCR. (B) The expression of circ_KIAA1199 in FHC cells and CRC cells (HCT116 and SW480) was detected by qRT-PCR. (C) QRT-PCR was used to detect circ_KIAA1199 expression in HCT16 and SW480 cells treated with different concentrations $(5,10$, and $20 \mu \mathrm{M})$ of curcumin. (D-E) Subcellular localization assay was performed to assess the localization of circ_KIAA1199 in the nucleus and cytoplasm of CRC cells. (F-G) ActD assay was used to evaluate the stability of circ_KIAA1199 in HCT16 and SW480 cells. ${ }^{\star} P<0.05$. 

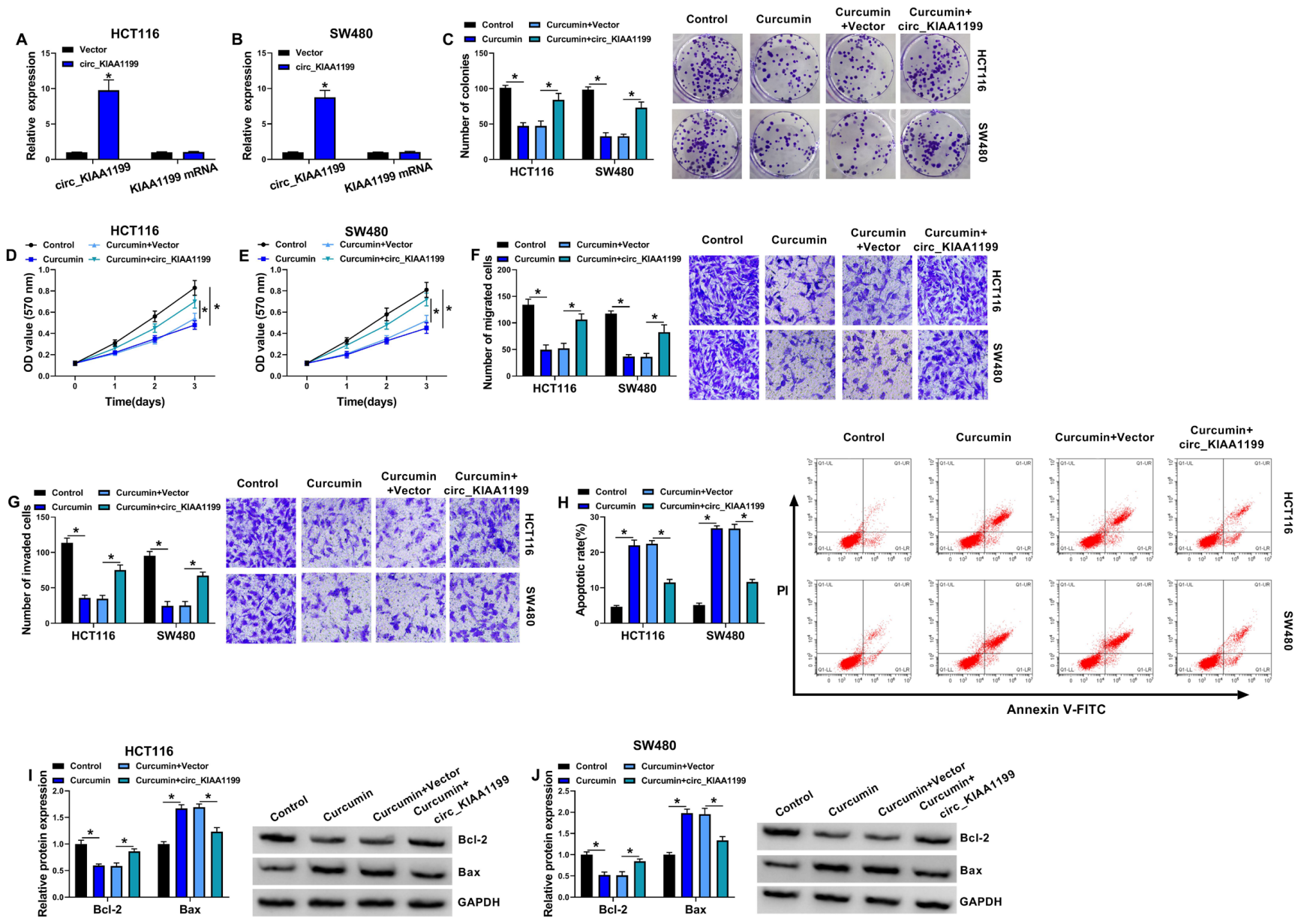

Figure 3. Curcumin regulated circ_KIAA1199 to mediate CRC progression. (A-B) After HCT16 and SW480 cells were transfected with circ_ KIAA1199 overexpression vector or vector, the expression of circ_KIAA1199 and linear KIAA1199 was measured by qRT-PCR. (C-J) HCT116 and SW480 cells were transfected with or without circ_KIAA1199 overexpression vector or vector, and then treated with curcumin. Non-transfected and non-treated cells were used as control. (C) Colony formation assay was performed to detect the number of colonies. (D-E) MTT assay was used to measure the cell viability. (F-G) The numbers of migrated and invaded cells were analyzed by transwell assay. (H) The apoptotic rate of cells was examined using flow cytometry. (I-J) The protein levels of Bcl-2 and Bax were determined using $\mathrm{WB}$ analysis. ${ }^{\star} P<0.05$.

\subsection{Circ_KIAA1199 targeted miR-433-3p}

The results that circ_KIAA1199 was mainly distributed in the cytoplasm suggested that circ_KIAA1199 might act as a miRNA sponge. To confirm the targeted miRNAs of circ_KIAA1199, we performed the bioinformatics analysis using circinteractome software (https://circinteractome.nia. nih.gov/). It was found that miR-433-3p had complementary binding sites with circ_KIAA1199 (Figure 4A). Further dualluciferase reporter assay results showed that miR-433-3p mimic reduced the luciferase activity of the circ_KIAA1199-WT vector, but it had no affect that of the circ_KIAA1199-MUT vector (Figure 4B-C), confirming the interaction between miR-433-3p and circ_KIAA1199. By detecting miR-433-3p expression, we discovered that miR-433-3p expression could be inhibited by circ_KIAA1199 overexpression (Figure 4D), while enhanced by curcumin (Figure 4E). Compared to FHC cells, miR-433-3p was discovered to be lowly expressed in HCT116 and SW480 cells (Figure 4F). Furthermore, a significantly downregulated miR-433-
$3 p$ was found in CRC tumor tissues (Figure $4 \mathrm{G}$ ), and correlation analysis showed that miR-433-3p expression was negatively correlated with circ_KIAA1199 expression in CRC tumor tissues (Figure 4H). All data showed that circ_KIAA1199 acted as miR433-3p sponge in CRC.

\subsection{Circ_KIAA1199 sponged miR-433-3p to participate in the regulation of curcumin on CRC progression}

To further determine whether circ_KIAA1199 sponged miR-433-3p during the process of curcumin regulating CRC progression, we co-transfected with circ_KIAA1199 overexpression vector and miR-433-3p mimic into HCT116 and SW480 cells. The detection results of miR-433-3p expression confirmed that the addition of miR-433-3p mimic could abolish the inhibiting effect of circ_KIAA1199 on miR-433-3p expression (Figure 5A). Then, the transfected cells were treated with curcumin. Function analysis results showed that miR-433-3p overexpression could 
A

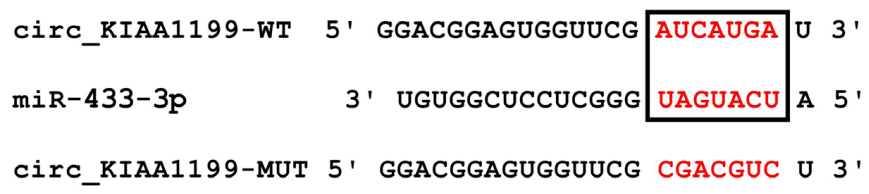

D

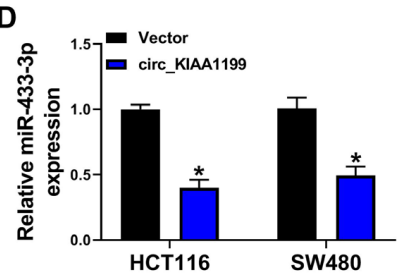

G

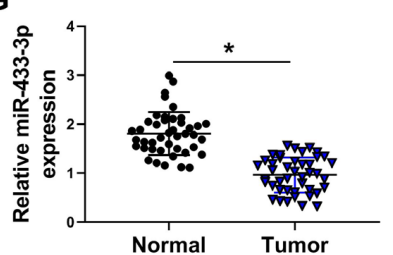

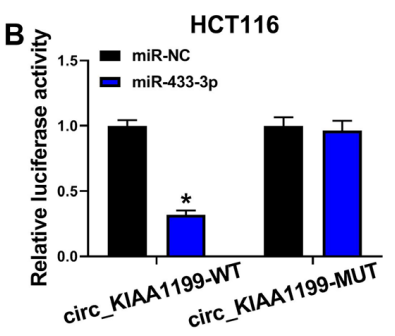

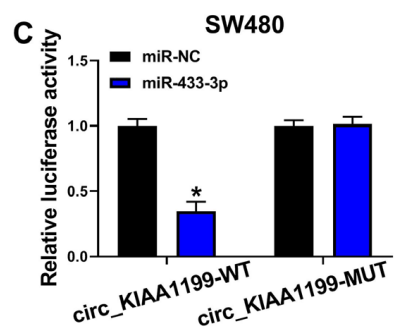

$\mathbf{F}$
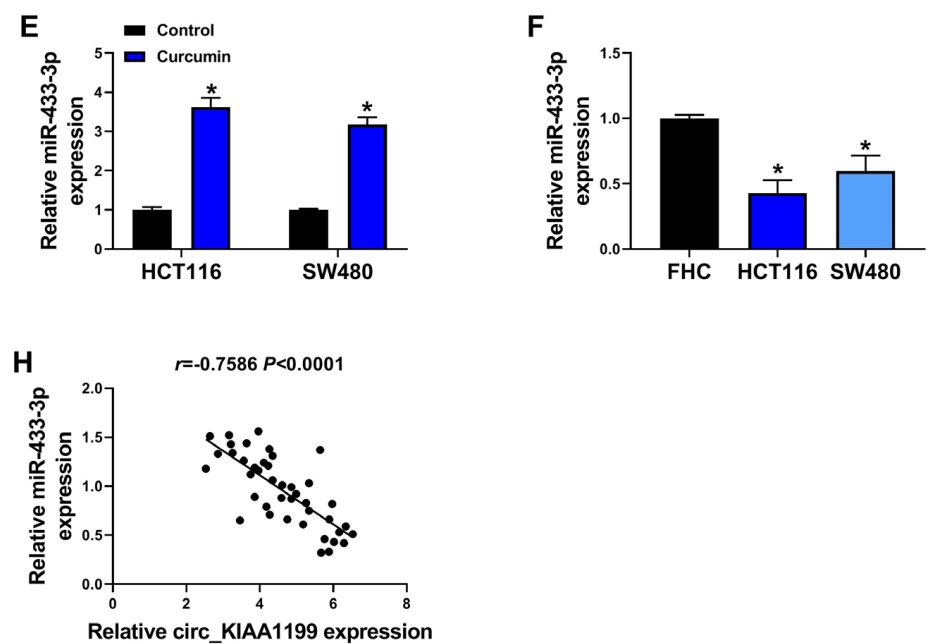

Figure 4. Circ_KIAA1199 targeted miR-433-3p. (A) The binding sites and corresponding mutate sites of circ_KIAA1199 and miR-433-3p were shown. (B-C) The interaction between circ_KIAA1199 and miR-433-3p was confirmed by dual-luciferase reporter assay. (D) The expression of miR-433-3p was measured by qRT-PCR in HCT116 and SW480 cells transfected with vector or circ_KIAA1199 overexpression vector. (E) QRT-PCR was used to detect miR-433-3p expression in HCT16 and SW480 cells treated with $20 \mu \mathrm{M}$ curcumin. (F) QRT-PCR was employed to examine the expression of miR-433-3p in FHC cells and CRC cells (HCT116 and SW480). (G) MiR-433-3p expression in CRC tumor tissues (Tumor) and adjacent normal tissues (Normal) were measured by qRT-PCR. (H) The correlation between circ_KIAA1199 and miR-433-3p was analyzed using Pearson correlation analysis. ${ }^{\star} P<0.05$.

reverse the promotion effect of circ_KIAA1199 on the number of colonies and cell viability, as well as the numbers of migrated and invaded cells in HCT116 and SW480 cells treated with curcumin (Figure 5B-F). Also, overexpressing of miR-433-3p reversed the apoptotic rate of curcumin-treated CRC cells inhibited by circ_KIAA1199 overexpression (Figure 5G). Moreover, the addition of miR-433-3p mimic also overturned the regulation of circ_KIAA1199 on the protein levels of Bcl-2 and Bax in curcumin-treated CRC cells, which could be observed by the decreased Bcl-2 level and the increased Bax level in the cotransfection group (Figure 5H, I). All data revealed that curcumin regulated CRC progression by regulating the circ_KIAA1199/ miR-433-3p axis.

\subsection{MiR-433-3p targeted ADAM10 in CRC}

MiRNA can usually bind to the 3'UTR of the target gene to cause mRNA degradation or transcription inhibition (Pu et al., 2019). Here, the Starbase3.0 software (http://starbase.sysu. edu.cn/) was used to predict the targets for miR-433-3p and ADAM10 3'UTR was found to can bound with miR-433-3p (Figure 6A). Moreover, we confirmed that only the luciferase activity of the ADAM10-WT vector could be inhibited by miR-433-3p mimic, which further confirmed that miR-433$3 p$ could interact with ADAM10 (Figure 6B, C). In order to determine the regulation of miR-433-3p on ADAM10, we overexpressed and knocked down the expression of miR-433-3p using its mimic and inhibitor (Figure 6D). Our data suggested that ADAM10 expression could be reduced by miR-433-3p overexpression and enhanced by miR-433-3p inhibition at the mRNA level and protein level (Figure 6E, F). Through detecting ADAM10 expression, we discovered that ADAM10 mRNA and protein expression was markedly higher in CRC tumor tissues than in adjacent normal tissues (Figure 6G, H), and its mRNA expression also was negatively correlated with miR-433-3p expression and positively correlated with circ_KIAA1199 expression in CRC tumor tissues (Figure 6I, J). Besides, the remarkably increased ADAM10 mRNA and protein expression also was detected in HCT116 and SW480 cells compared with that in FHC cells (Figure 6K, L). In addition, we found that curcumin had an inhibition effect on the mRNA and protein expression of ADAM10 (Figure 6M, N), which was consistent with the effect of curcumin on circ_KIAA1199 expression. In HCT116 and SW480 cells transfected with circ-KIAA1199 overexpression 

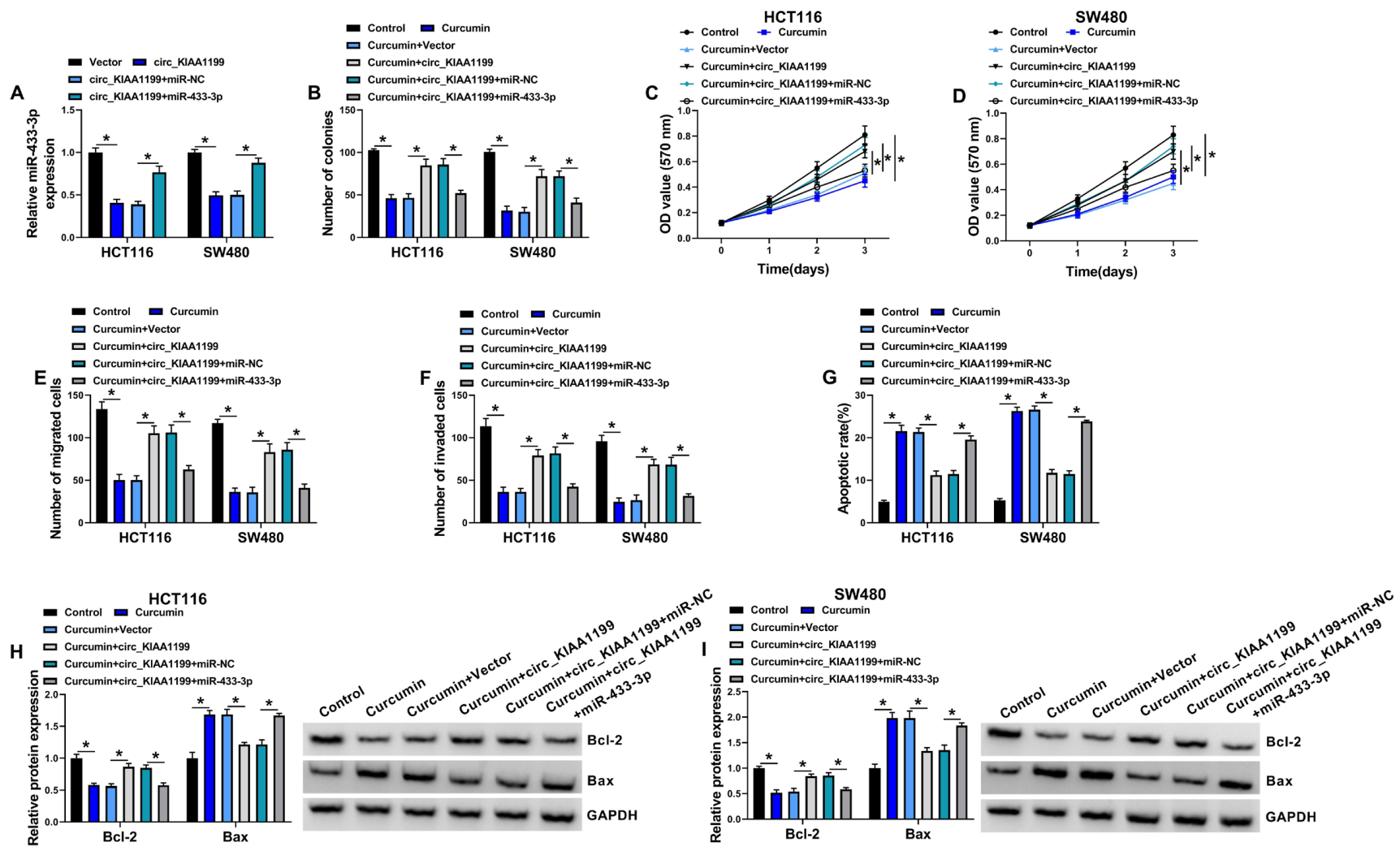

Figure 5. Curcumin regulated CRC progression by regulating circ_KIAA1199/miR-433-3p. (A) HCT16 and SW480 cells were transfected with vector, circ_KIAA1199, circ_KIAA1199 + miR-NC or circ_KIAA1199 + miR-433-3p. The expression of miR-433-3p was detected by qRT-PCR. (B-I) After transfection, HCT116 and SW480 cells were treated with curcumin. Non-transfected and non-treated cells were used as control. (B) The number of colonies was examined using colony formation assay. (C-D) MTT assay was employed to determine the cell viability. (E-F) Transwell assay was performed to test the numbers of migrated and invaded cells. (G) The apoptotic rate of cells was determined by flow cytometry. (H-I) $\mathrm{WB}$ analysis was utilized for examining the protein levels of Bcl-2 and Bax. ${ }^{\star} P<0.05$.

vector and miR-433-3p mimic, we uncovered that circ KIAA1199 could promote ADAM10 expression, and this effect could be abolished by miR-433-3p overexpression (Figure 6O, P). Our data illuminated that circ_KIAA1199 sponged miR-433-3p to regulate ADAM10 in CRC.

\subsection{ADAM10 silencing reversed the regulation of $m i R-433-$ $3 p$ inhibitor on the progression of curcumin-treated CRC cells}

After that, anti-miR-433-3p and si-ADAM10 were cotransfected into HCT116 and SW480 cells to perform function experiments for confirming the existed miR-433-3p/ADAM10 axis in CRC. The si-ADAM10 could abolish the promoting effect of miR-433-3p inhibitor on ADAM10 mRNA and protein expression, indicating that the transfection was successful (Figure 7A, B). Under the treatment with curcumin, we discovered that miR433-3p inhibitor could enhance the number of colonies, the cell viability, and the numbers of migrated and invaded cells in curcumin-treated CRC cells. However, the addition of siADAM10 could reverse the regulation of miR-433-3p on the proliferation, migration and invasion of curcumin-treated
CRC cells (Figure 7C-G). Also, knockdown of ADAM10 also abolished the suppressive effect of miR-433-3p inhibitor on the apoptotic rate of HCT116 and SW480 cells treated with curcumin (Figure 7H). Meanwhile, miR-433-3p inhibitor promoted the $\mathrm{Bcl}-2$ protein level and reduced the Bax protein level in curcumin-treated CRC cells, while ADAM10 silencing also could overturn these effects (Figure 7I, J). Hence, all results suggested that miR-433-3p targeted ADAM10 to be involved in the regulation of curcumin on CRC progression.

\subsection{Circ_KIAA1199 abolished the reduction of curcumin on CRC tumor growth in vivo}

Animal experiments were performed to further confirm the role of curcumin on CRC tumor growth. The detection results of tumor volume and tumor weight showed that curcumin treatment could markedly reduce the tumor volume and tumor weight of mice. However, the tumor volume and tumor weight of mice in the curcumin + lenti-circ_KIAA1199 group were significantly enhanced compared to the curcumin + lenti-NC group (Figure 8A, B). In the tumor tissues of mice, we discovered that the circ_KIAA1199 expression was decreased, miR-433-3p 
A

ADAM10 3 ' UTR

ADAM10-WT 5' AUACUACUUCAGAGU UCAUGA U 3'

miR-433-3p 3' UGUGGCUCCUCGGGU AgUACU A 5 '

ADAM10-MUT 5' AUACUACUUCAgAgU GacGUC U 3
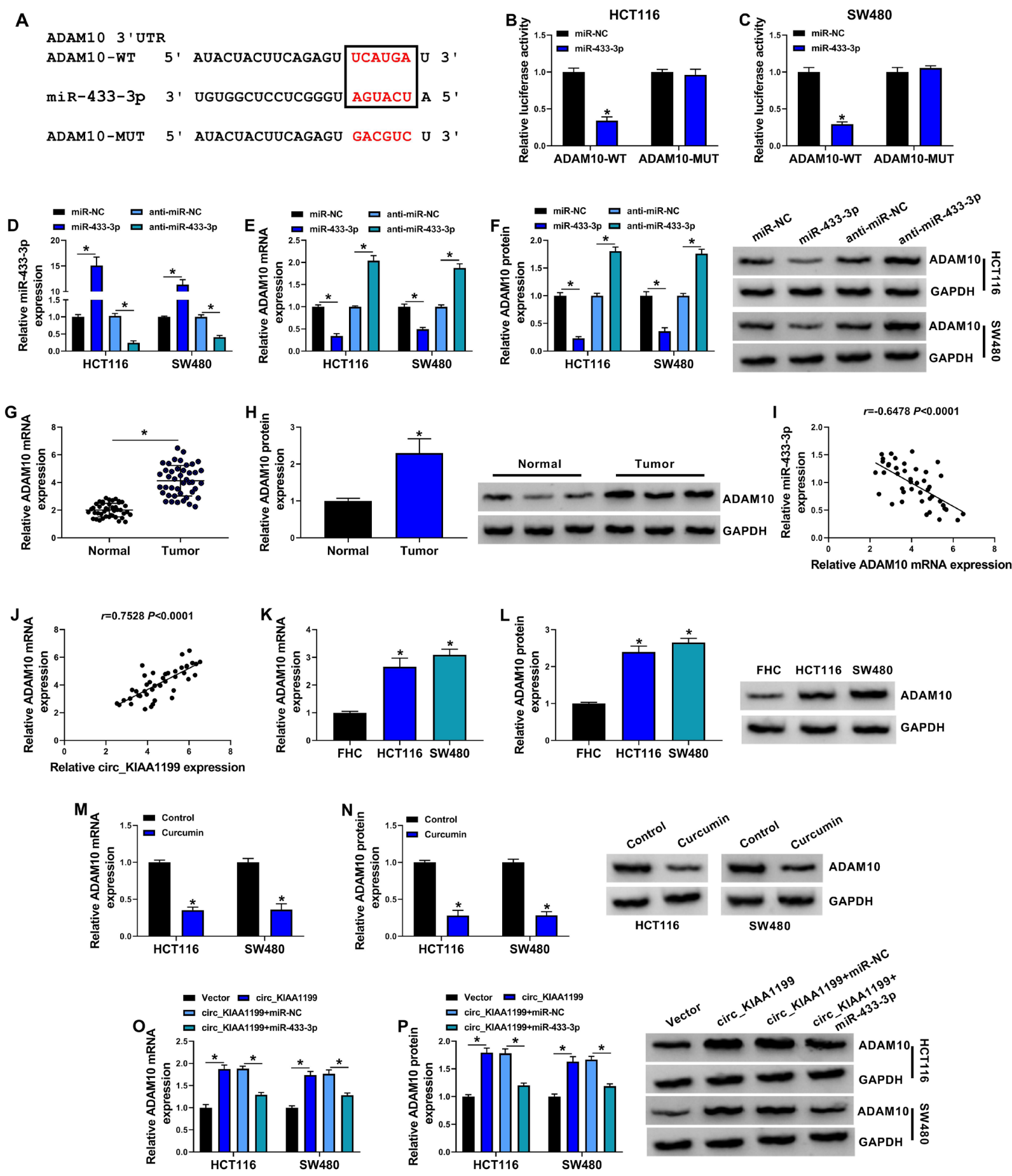

Figure 6. MiR-433-3p targeted ADAM10 in CRC. (A) The binding sites and corresponding mutate sites of miR-433-3p and ADAM10 3'UTR were shown. (B-C) Dual-luciferase reporter assay was utilized for assessing the interaction between miR-433-3p and ADAM10. (D) The transfection efficiencies of miR-433-3p mimic and inhibitor were confirmed by detecting miR-433-3p expression using qRT-PCR. (E-F) The mRNA and protein expression levels of ADAM10 were measured by qRT-PCR and WB analysis in HCT116 and SW480 cells transfected with miR-NC, miR-433-3p, anti-miR-NC or anti-miR-433-3p. (G-H) The mRNA and protein expression of ADAM10 in CRC tumor tissues (Tumor) and adjacent normal tissues (Normal) were determined using qRT-PCR and WB analysis. (I-J) Pearson correlation analysis was utilized for evaluating the correlation between ADAM10 mRNA expression and miR-433-3p or circ_KIAA1199 expression in CRC tumor tissues. (K-L) QRT-PCR and WB analysis were employed to examine the mRNA and protein expression of ADAM10 in FHC cells and CRC cells (HCT116 and SW480). (M-N) QRT-PCR and WB analysis were used to detect the mRNA and protein expression of ADAM10 in HCT16 and SW480 cells treated with $20 \mu \mathrm{M}$ curcumin. (O-P) HCT116 and SW480 cells were transfected with vector, circ_KIAA1199, circ_KIAA1199 + miR-NC or circ_KIAA1199 + miR-433-3p. The mRNA and protein expression levels of ADAM10 were examined by qRT-PCR and WB analysis. ${ }^{\star} P<0.05$. 

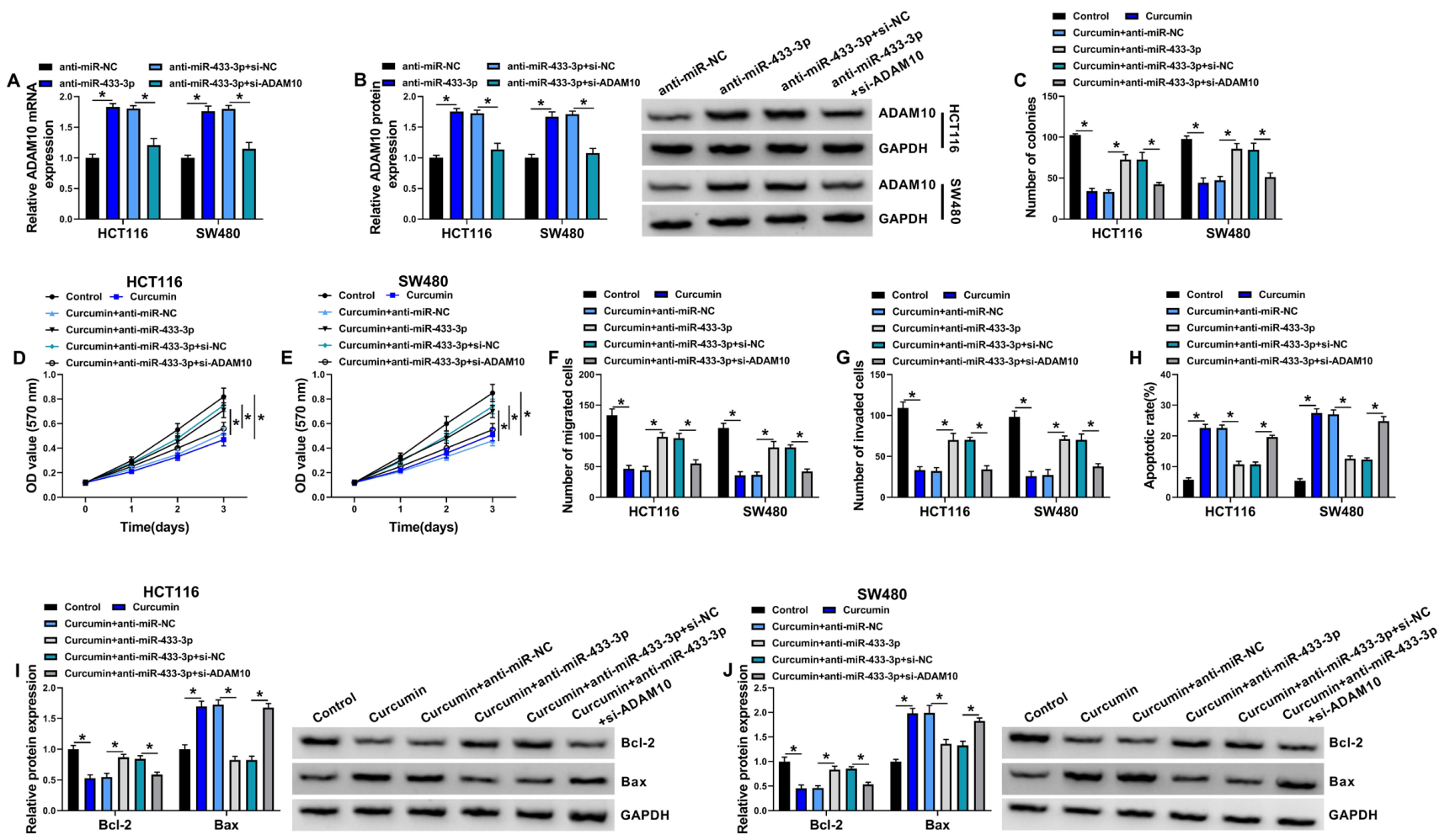

Figure 7. Curcumin inhibited CRC progression via the miR-433-3p/ADAM10 axis. (A-B) HCT16 and SW480 cells were transfected with antimiR-NC, anti-miR-433-3p, anti-miR-433-3p + si-NC or anti-miR-433-3p + si-ADAM10. The mRNA and protein expression of ADAM10 was detected by qRT-PCR and WB analysis. (C-J) After transfection, HCT116 and SW480 cells were treated with curcumin. Non-transfected and non-treated cells were used as control. (C) Colony formation assay was employed to measure the number of colonies. (D-E) Cell viability was tested using MTT assay. (F-G) Transwell assay was utilized to assess the numbers of migrated and invaded cells. (H) Flow cytometry was used to detect the apoptotic rate of cells. (I-J) The protein levels of Bcl-2 and Bax were tested using WB analysis. ${ }^{\star} P<0.05$.

was increased, and ADAM10 mRNA and protein expression was inhibited in the curcumin treatment group, while these effects were eliminated in the curcumin + lenti-circ_KIAA1199 group (Figure 8C-F). In addition, IHC staining revealed that the Ki67 positive cells was decreased and Cleaved caspase 3 positive cells was enhanced in the tumor tissues of the curcumin group, and circ_KIAA1199 overexpression could reverse these effects (Figure 8G). Therefore, we confirmed that curcumin could reduce CRC tumor growth by regulating the circ_KIAA1199/ miR-433-3p/ADAM10 pathway.

\section{Discussion}

A large number of studies have confirmed that curcumin can regulate anti-tumor molecule targets in cancer (Pandya et al., 2021), which is expected to achieve a breakthrough in the research of natural targeted drugs. Curcumin can not only inhibit the proliferation and metastasis, but also attenuate the chemotherapy resistance of cancer cells (Su et al., 2018). In this study, we confirmed that curcumin indeed suppressed CRC cell proliferation, metastasis and accelerated apoptosis in vitro, and inhibited tumor growth in vivo. CircRNA is a key regulator of cancer progression and has been widely demonstrated as a potential molecular target for cancer therapy (Kristensen et al.,
2018). Studies have shown that curcumin may regulate the radio-sensitization of nasopharyngeal carcinoma through mediating circRNA network (Yang et al., 2020; Zhu et al., 2020). Unfortunately, it is unclear whether curcumin can participate in CRC progression by regulating the circRNA network.

Tian et al. screened the differentially expressed circRNAs in CRC tissues and normal tissues by microarray analysis, and uncovered that circ_KIAA1199 was overexpressed in CRC tissues (Tian et al., 2019). Here, we verified the highly circ_KIAA1199 expression in CRC tissues and cells. To our surprise, we found that curcumin could inhibit circ_KIAA1199 expression. Around the hypothesis that curcumin might mediate the circ_KIAA1199 network, we conducted out the circ_KIAA1199 overexpression experiment in curcumin-treated CRC cells. The results indicated that the regulation of curcumin on CRC proliferation, metastasis and apoptosis could be abolished by overexpressing circ_KIAA1199. Additionally, in vivo experiments also revealed that the antitumorigenesis role of curcumin in CRC also could be reversed by circ_KIAA1199 overexpression. These results confirmed that curcumin indeed mediated the progression of CRC via regulating circ_KIAA1199.

It has been widely demonstrated that circRNAs function as molecular sponges of miRNAs (Song et al., 2020; Wu et al., 2019). 

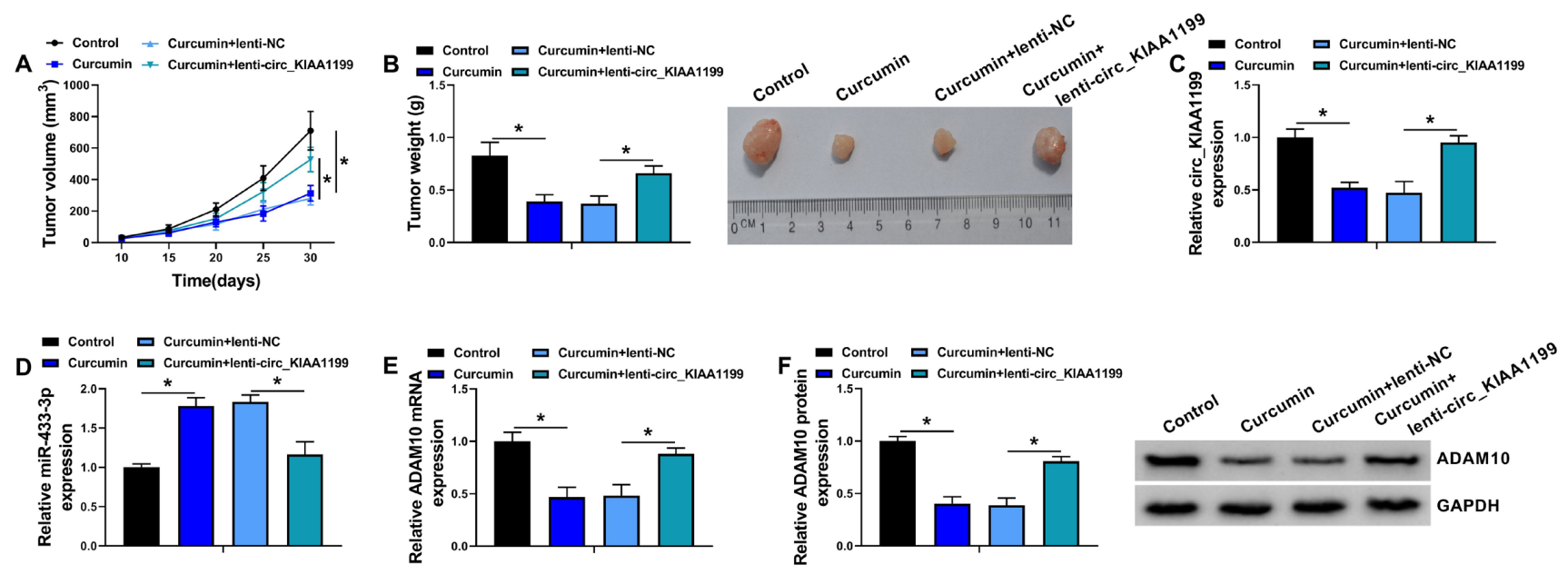

G
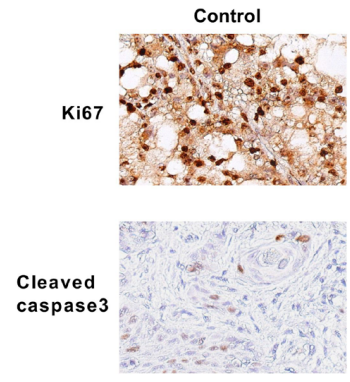
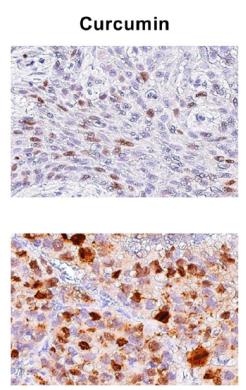
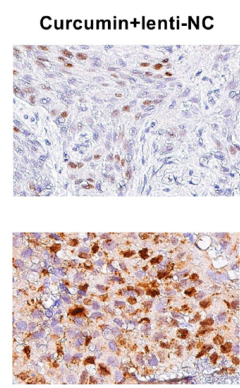
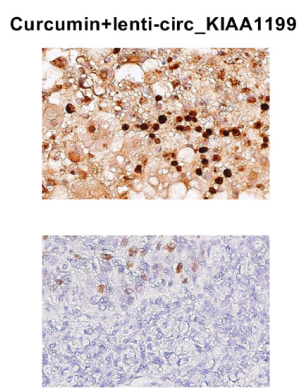

Figure 8. Curcumin reduced CRC tumor growth through regulating the circ_KIAA1199/miR-433-3p/ADAM10 axis in vivo. Tumor volume (A) and tumor weight (B) were detected in each group. (C-E) The expression levels of circ_KIAA1199, miR-433-3p and ADAM10 in the tumor tissues of each group were measured by qRT-PCR. (F) The ADAM10 protein level in the tumor tissues of each group was determined by WB analysis. (G) IHC staining was used to detect the Ki67 and Cleaved caspase3 positive cells to assess the proliferation and apoptosis of tumor cells. ${ }^{\star} P<0.05$.

Through bioinformatics prediction and dual-luciferase reporter assay verification, we discovered that circ_KIAA1199 could interact with miR-433-3p. MiR-433-3p is underexpressed in many types of cancer and has been shown to play tumor suppressive effects in cancers, such as esophageal squamous cell carcinoma (Shi et al., 2018), bladder cancer (Wang et al., 2020), and renal carcinoma (Cai et al., 2020). In CRC-related studies, low miR433 expression was considered to be a marker of early relapse in CRC patients, and it played an anti-tumor role in CRC by inhibiting cell proliferation and metastasis (Li et al., 2017; Zhang et al., 2018b). In this, we found that miR-433-3p expression could be promoted by curcumin. The rescue experiments revealed that the enhancing effect of circ_KIAA1199 on the progression of curcumin-treated CRC cells could be reversed by miR-433-3p. All data suggested that circ_KIAA1199 could act as miR-433-3p sponge to be involved in the regulation of curcumin on CRC progression.

MiR-433-3p was found to target CCAR1, FGF2, and GRB2 to regulate cancer development (Cai et al., 2020; Shi et al., 2018; Wang et al., 2020). Here, we discovered that ADAM10 was a newly target of miR-433-3p. ADAM10 is a member of ADAM family with proteolytic enzyme activity (Seegar et al., 2017). Studies have shown that ADAM10 regulates the process of many physiological processes such as cell signal transduction, extracellular matrix degradation, and cell adhesion by binding with cell membranes (Toonen et al., 2016; White, 2003). At the same time, ADAM10 has also been confirmed to exert an important function in cancer development (Tsang et al., 2018). In past studies, ADAM10 was proved to be overexpressed in CRC and to promote CRC malignant progression (Hong et al., 2020; Sun et al., 2019). Here, we uncovered that circ_KIA1199 could sponge miR-433-3p to positively regulate ADAM10. Besides, the reversal effect of ADAM10 silencing on miR-433-3p inhibitor regulating the progression of curcumin-treated CRC cells confirmed that the miR-433-3p/ADAM10 axis taken part in the regulation of curcumin on CRC progression.

In conclusion, our data showed that curcumin could suppress CRC proliferation and metastasis through regulating the circ_KIAA1199/miR-433-3p/ADAM10 axis. Our study not only uncovered the new discovery that curcumin mediated CRC progression through the circRNA axis, but also revealed the role and mechanism of a novel circRNA, circ_KIAA1199, in CRC. These results provided a theoretical basis for us to better understand the anti-cancer effect of curcumin.

\section{Conflicts of interest}

The authors have no conflict of interest to declare. 


\section{Funding}

None.

\section{Reference}

Brody, H. (2015). Colorectal cancer. Nature, 521(7551), S1. http:// dx.doi.org/10.1038/521S1a. PMid:25970450.

Cai, X., Zhang, X., Mo, L., Zhu, J., \& Yu, H. (2020). LncRNA PCGEM1 promotes renal carcinoma progression by targeting miR-433-3p to regulate FGF2 expression. Cancer Biomarkers, 27(4), 493-504. http:// dx.doi.org/10.3233/CBM-190669. PMid:31958075.

Danciu, C. (2020). Natural bioactive compounds, vegetal extracts and modern pharmaceutical formulations: new insights into the anti-cancer mechanism of action. Anti-cancer Agents in Medicinal Chemistry, 20(15), 1754-1755. http://dx.doi.org/10.2174/1871520 62015200911152012 . PMid:33138743.

Hao, Q., \& Zhang, Z. (2020). hsa_circRNA_000166 facilitated cell growth and limited apoptosis through targeting miR-326/LASP1 axis in colorectal cancer. Gastroenterology Research and Practice, 2020, 8834359. http://dx.doi.org/10.1155/2020/8834359. PMid:33376485.

Hong, Y. G., Xin, C., Zheng, H., Huang, Z. P., Yang, Y., Zhou, J. D., Gao, X. H., Hao, L., Liu, Q. Z., Zhang, W., \& Hao, L. Q. (2020). miR-365a$3 p$ regulates ADAM10-JAK-STAT signaling to suppress the growth and metastasis of colorectal cancer cells. Journal of Cancer, 11(12), 3634-3644. http://dx.doi.org/10.7150/jca.42731. PMid:32284760.

Kotha, R. R., \& Luthria, D. L. (2019). Curcumin: biological, pharmaceutical, nutraceutical, and analytical aspects. Molecules, 24(16), 2930. http:// dx.doi.org/10.3390/molecules24162930. PMid:31412624.

Kristensen, L. S., Hansen, T. B., Veno, M. T., \& Kjems, J. (2018). Circular RNAs in cancer: opportunities and challenges in the field. Oncogene, 37(5), 555-565. http://dx.doi.org/10.1038/onc.2017.361. PMid:28991235.

Lei, B., Tian, Z., Fan, W., \& Ni, B. (2019). Circular RNA: a novel biomarker and therapeutic target for human cancers. International Journal of Medical Sciences, 16(2), 292-301. http://dx.doi.org/10.7150/ ijms.28047. PMid:30745810.

Li, J., Mao, X., Wang, X., Miao, G., \& Li, J. (2017). miR-433 reduces cell viability and promotes cell apoptosis by regulating MACC1 in colorectal cancer. Oncology Letters, 13(1), 81-88. http://dx.doi. org/10.3892/ol.2016.5445. PMid:28123526.

Li, W., Li, C., Zheng, H., Chen, G., \& Hua, B. (2016). Therapeutic targets of Traditional Chinese Medicine for colorectal cancer. Journal of Traditional Chinese Medicine, 36(2), 243-249. http://dx.doi. org/10.1016/S0254-6272(16)30034-6. PMid:27400481.

Nelson, K. M., Dahlin, J. L., Bisson, J., Graham, J., Pauli, G. F., \& Walters, M. A. (2017). The essential medicinal chemistry of curcumin. Journal of Medicinal Chemistry, 60(5), 1620-1637. http://dx.doi.org/10.1021/ acs.jmedchem.6b00975. PMid:28074653.

Pandya, N., Khan, E., Jain, N., Satham, L., Singh, R., Makde, R. D., Mishra, A., \& Kumar, A. (2021). Curcumin analogs exhibit anticancer activity by selectively targeting G-quadruplex forming c-myc promoter sequence. Biochimie, 180, 205-221. http://dx.doi. org/10.1016/j.biochi.2020.11.006. PMid:33188859.

Patel, S. G., \& Ahnen, D. J. (2018). Colorectal cancer in the young. Current Gastroenterology Reports, 20(4), 15. http://dx.doi.org/10.1007/ s11894-018-0618-9. PMid:29616330.

Pricci, M., Girardi, B., Giorgio, F., Losurdo, G., Ierardi, E., \& Di Leo, A. (2020). Curcumin and colorectal cancer: from basic to clinical evidences. International Journal of Molecular Sciences, 21(7), 2364. http://dx.doi.org/10.3390/ijms21072364. PMid:32235371.
Pu, M., Chen, J., Tao, Z., Miao, L., Qi, X., Wang, Y., \& Ren, J. (2019). Regulatory network of miRNA on its target: coordination between transcriptional and post-transcriptional regulation of gene expression. Cellular and Molecular Life Sciences, 76(3), 441-451. http://dx.doi. org/10.1007/s00018-018-2940-7. PMid:30374521.

Seegar, T. C. M., Killingsworth, L. B., Saha, N., Meyer, P. A., Patra, D., Zimmerman, B., Janes, P. W., Rubinstein, E., Nikolov, D. B., Skiniotis, G., Kruse, A. C., \& Blacklow, S. C. (2017). Structural Basis for Regulated Proteolysis by the $\alpha$-Secretase ADAM10. Cell, 171(7), 1638-1648.

Sheng, S., Zhao, T., \& Wang, X. (2018). Comparison of robot-assisted surgery, laparoscopic-assisted surgery, and open surgery for the treatment of colorectal cancer: a network meta-analysis. Medicine, 97(34), e11817. http://dx.doi.org/10.1097/MD.0000000000011817. PMid:30142771.

Shi, Q., Wang, Y., Mu, Y., Wang, X., \& Fan, Q. (2018). MiR-433-3p inhibits proliferation and invasion of esophageal squamous cell carcinoma by targeting GRB2. Cellular Physiology and Biochemistry, 46(5), 2187-2196. http://dx.doi.org/10.1159/000489548. PMid:29730656.

Song, H., Sun, J., Kong, W., Ji, Y., Xu, D., \& Wang, J. (2020). Construction of a circRNA-Related ceRNA prognostic regulatory network in breast cancer. OncoTargets and Therapy, 13, 8347-8358. http:// dx.doi.org/10.2147/OTT.S266507. PMid:32922032.

Su, P., Yang, Y., Wang, G., Chen, X., \& Ju, Y. (2018). Curcumin attenuates resistance to irinotecan via induction of apoptosis of cancer stem cells in chemoresistant colon cancer cells. International Journal of Oncology, 53(3), 1343-1353. http://dx.doi.org/10.3892/ijo.2018.4461. PMid:29956726.

Sun, S. Q., Ren, L. J., Liu, J., Wang, P., \& Shan, S. M. (2019). Sevoflurane inhibits migration and invasion of colorectal cancer cells by regulating microRNA-34a/ADAM10 axis. Neoplasma, 66(6), 887-895. http:// dx.doi.org/10.4149/neo_2018_181213N962. PMid:31305122.

Tian, J., Xi, X., Wang, J., Yu, J., Huang, Q., Ma, R., Zhang, X., Li, H., \& Wang, L. (2019). CircRNA hsa_circ_0004585 as a potential biomarker for colorectal cancer. Cancer Management and Research, 11, 54135423. http://dx.doi.org/10.2147/CMAR.S199436. PMid:31354349.

Toonen, J. A., Ronchetti, A., \& Sidjanin, D. J. (2016). A Disintegrin and Metalloproteinase10 (ADAM10) regulates NOTCH signaling during early retinal development. PLoS One, 11(5), e0156184. http://dx.doi. org/10.1371/journal.pone.0156184. PMid:27224017.

Tsang, J. Y. S., Lee, M. A., Chan, T. H., Li, J., Ni, Y. B., Shao, Y., Chan, S. K., Cheungc, S. Y., Lau, K. F., \& Tse, G. M. K. (2018). Proteolytic cleavage of amyloid precursor protein by ADAM10 mediates proliferation and migration in breast cancer. EBioMedicine, 38, 8999. http://dx.doi.org/10.1016/j.ebiom.2018.11.012. PMid:30470613.

Unlu, A., Nayir, E., Kalenderoglu, M. D., Kirca, O., \& Ozdogan, M. (2016). Curcumin (Turmeric) and cancer. Journal of the Balkan Union of Oncology, 21(5), 1050-1060. PMid:27837604.

Wang, F., Fan, M., Cai, Y., Zhou, X., Tai, S., Yu, Y., Wu, H., Zhang, Y., Liu, J., Huang, S., He, N., Hu, Z., \& Jin, X. (2020). Circular RNA circRIMS1 acts as a sponge of miR-433-3p to promote bladder cancer progression by regulating CCAR1 expression. Molecular Therapy. Nucleic Acids, 22, 815-831. http://dx.doi.org/10.1016/j. omtn.2020.10.003. PMid:33230478.

Wang, G. R., Wang, Z. W., \& Jin, Z. Y. (2019). Application and progress of texture analysis in the therapeutic effect prediction and prognosis of neoadjuvant chemoradiotherapy for colorectal cancer. Chinese Medical Sciences Journal, 34(1), 45-50. http://dx.doi.org/10.24920/003572. PMid:30961780. 
Weitz, J., Koch, M., Debus, J., Hohler, T., Galle, P. R., \& Buchler, M. W. (2005). Colorectal cancer. Lancet, 365(9454), 153-165. http://dx.doi. org/10.1016/S0140-6736(05)17706-X. PMid:15639298.

White, J. M. (2003). ADAMs: modulators of cell-cell and cell-matrix interactions. Current Opinion in Cell Biology, 15(5), 598-606. http:// dx.doi.org/10.1016/j.ceb.2003.08.001. PMid:14519395.

Wu, J., Liu, S., Xiang, Y., Qu, X., Xie, Y., \& Zhang, X. (2019). Bioinformatic analysis of circular RNA-associated ceRNA network associated with hepatocellular carcinoma. BioMed Research International, 2019, 8308694. http://dx.doi.org/10.1155/2019/8308694. PMid:31886256.

Wu, M., Kong, C., Cai, M., Huang, W., Chen, Y., Wang, B., \& Liu, X. (2020). Hsa_circRNA_002144 promotes growth and metastasis of colorectal cancer through regulating miR-615-5p/LARP1/mTOR pathway. Carcinogenesis, 42(4), 601-610.

Xu, H., Liu, Y., Cheng, P., Wang, C., Liu, Y., Zhou, W., Xu, Y., \& Ji, G. (2020). CircRNA_0000392 promotes colorectal cancer progression through the miR-193a-5p/PIK3R3/AKT axis. Journal of Experimental \& Clinical Cancer Research, 39(1), 283. http://dx.doi.org/10.1186/ s13046-020-01799-1. PMid:33317596.

Yang, J., Zhu, D., Liu, S., Shao, M., Liu, Y., Li, A., Lv, Y., Huang, M., Lou, D., \& Fan, Q. (2020). Curcumin enhances radiosensitization of nasopharyngeal carcinoma by regulating circRNA network. Molecular Carcinogenesis, 59(2), 202-214. http://dx.doi.org/10.1002/ mc.23143. PMid:31793078.

Zhang, H. D., Jiang, L. H., Sun, D. W., Hou, J. C., \& Ji, Z. L. (2018a). CircRNA: a novel type of biomarker for cancer. Breast Cancer, 25(1), 1-7. http://dx.doi.org/10.1007/s12282-017-0793-9. PMid:28721656.

Zhang, J., Zhang, L., Zhang, T., Dong, X. M., Zhu, Y., \& Chen, L. H. (2018b). Reduced miR-433 expression is associated with advanced stages and early relapse of colorectal cancer and restored miR-433 expression suppresses the migration, invasion and proliferation of tumor cells in vitro and in nude mice. Oncology Letters, 15(5), 7579-7588. http://dx.doi.org/10.3892/ol.2018.8275. PMid:29740483.

Zhang, Z., Yang, T., \& Xiao, J. (2018c). Circular RNAs: promising biomarkers for human diseases. EBioMedicine, 34, 267-274. http:// dx.doi.org/10.1016/j.ebiom.2018.07.036. PMid:30078734.

Zhu, D., Shao, M., Yang, J., Fang, M., Liu, S., Lou, D., Gao, R., Liu, Y., Li, A., Lv, Y., Mo, Z., \& Fan, Q. (2020). Curcumin enhances radiosensitization of nasopharyngeal carcinoma via mediating regulation of tumor stem-like cells by a CircRNA network. Journal of Cancer, 11(8), 2360-2370. http://dx.doi.org/10.7150/jca.39511. PMid:32127962. 